\title{
Structural Health Monitoring and Damage Detection through Machine Learning approaches
}

\author{
Priyanka Singh*, Umaid Faraz Ahmad, and Siddharth Yadav \\ Department of Civil Engineering, Amity School of Engineering \& Technology, Amity University Uttar Pradesh, Noida, India
}

\begin{abstract}
Data-driven approaches are gaining popularity in structural health monitoring (SHM) due to recent technological advances in sensors, high-speed Internet and cloud computing. Since Machine learning (ML), particularly in SHM, was introduced in civil engineering, this modern and promising method has drawn significant research attention. SHM's main goal is to develop different data processing methodologies and generate results related to the different levels of damage recognition process. SHM implements a technique for damage detection and classification, including data from a system collected under different structural states using a piezoelectric sensor network using guided waves, hierarchical non-linear primary component analysis and machine learning. The primary objective of this paper is to analyse the current SHM literature using evolving ML-based methods and to provide readers with an overview of various SHM applications. The technique and implementation of vibration-based, vision-based surveillance, along with some recent SHM developments are discussed.
\end{abstract}

\section{Introduction}

Civil structures, including large bridges, dams, and highrise buildings, are becoming vulnerable to loss of serviceability as they fall apart from use. This inescapable loop needs severe maintenance [1-3]. Onsite studies, despite all, include the closing of bridges or the construction of buildings to examine them, due to humane constraints. Many researchers have suggested systemic health monitoring (SHM) procedures along these lines. SHM is an incredibly new technology that has grown over the last decades. Structural Health Monitoring (SHM) is one of the main applications for new sensor growth. The ability to discern damage at the start stage can reduce the costs and time associated with the fixing of basic damages. Predicting or detecting the onset of hazardous structural actions by observing longterm deterioration Is the main function of SHM. Infrastructures, due to the structural failure and loads caused by earthquakes, waves, automobiles or ambient vibrations, are vulnerable to significant physical damage. In the last few decades of practising engineers and scholars, safety and the need to lower cost of inspection have been ensured of high priority. Consequently, several forums stress the value of economic systemic health surveillance (ESM) to ensure long term structural stability and safety [5-7]. Different forms of modern SHM technologies can simplify frequent inspections and decreasing the direct and indirect costs associated with unnecessary ageing fails (use-echo impact, ultrasound surface waves, soil penetrating radar, electric resistance) [8-10] in addition to traditional inspections and non- destructive tests. Any SHM method and framework is based on sensors and sensor data (observable responses).

\section{Structural Health Monitoring and machine Learning}

Recent advancements in sensor technologies and networking (contact, cellular, etc.) have provided an unparalleled speed and ability for acquiring data. Furthermore, innovations have been utilised in diverse ways in other hardware and software support also. For example, the advancement of emerging infrastructure repair technology and the application of possible solutions, such as the Unmanned aerial systems (UAS), was a major focus. The latest UAS technology focuses on fully autonomous, long-distance multi-rotor systems with a large variety of SHM sensors. The sensors may include visible-spectrum light cameras, infrared and infrared cameras, radar systems and sonar systems with high-resolution applications. At least, a drone team inspect on the ground a bridge for human defects [8-10]. The power and data transmission requirements have traditionally prohibited the implementation of large sensor arrays on public networks, with the capacities and complexities relevant to the implementation of captive systems. In general, SHM methods focused on tested multi-physical models cannot be adapted to active models.

The calculated data need the least noise for simulations, which is not viable for functional conditions and systems. Therefore, models powered by data is demonstrated by flexibility. Machine learning (ML) is a

\footnotetext{
* Corresponding author: priyanka24978@gmail.com
} 
subcategory of artificial intelligence (AI). The ML techniques are focused to create workable algorithms that can benefit from the available estimated or simulated algorithms to make potential predictions [11 15]. ML methods are much broader as compared to other fields of computer engineering, whereas its applications in SHM are discussed in this study. The ML-based SHM models are specifically designed to learn on its own. ML-based SHM models can be categorised as supervised, unsupervised or enhanced learning. In supervised learning, an ML model can be trained while using a set of training data. If the effects of the algorithm are independent or categorical variables, the model is categorised as classification (i.e. vector support, neighbour k-near, Bayesian, decision-making, deep learning) or regression (e.g. neural networks, decisionmaking process, linear, non-linear regression) [16-20]. In unsupervised learning, there is no provision of training of dataset. Examples of unsupervised learning are Hierarchical clustering, partitional clustering, k-means, spectral clustering, etc. In reinforcement learning, the ideal performance is determined through agents, by try and error. It is interesting that the use of standard models requires expertise and functionality design skills for complex SHM applications, which are often not possible as tracked devices get more sophisticated due to their highly non-linear behaviour.

\section{Techniques for Structural Health Monitoring}

Structural health monitoring using vibration are based on the detection, location, classification, assessment, and prediction known as five levels of (SHM). The two major structural damage classifications are linear and non-linear. A linear-elastic structure will exist as the same, where modal characteristics and variations can be seen in a linear equation due to geometric or materialistic changes.

On the other hand, non-linear damage occurs if, after damage, a linear-elastic pattern or structure becomes non-linear at first. For example, developing a wear and tear crack can open and close under vibration under normal operating conditions, is an appropriate example of nonlinear damage. In general, a successful damage detection technique applies to both types of damage [2123]. As previously discussed, the SHM method involves several steps. First and foremost, the system is monitored over time using a variety of sensors and results are obtained from standard dynamic response measurement samples from the same sensors. The extraction of the characteristics is the next step in which these measurements derive the characteristics which can cause damage. Further statistical analysis is carried out on these receptive characteristics in order to assess the present circumstances and the health of the structure. In the case of long-term systematic monitoring situations, the statistical process output is regularly revised.

Acquiring information to validate the structural potential smoothly as it is subjected to ageing and deterioration due to various environmental conditions is possible through SHM. Furthermore, SHM is an emergency aid to track the functional integrity of the structure if the device has adverse effects due to events such as earthquakes and heavy loadings. The advances in Wireless Sensor Networks (WSNs) have influenced the SHM technology, enabling wireless, particularly through remote access, transmission of monitoring parameters. Figure 1 shows the structural dynamic responses through (WSNs).

In the case of aircraft structure and buildings SHM is widely used for detecting damage in real time in the whole structural system [24-25]. Damage detection method also consists of Condition Monitoring [CM] which is similar to SHM, but it is used in the calculation of damage to SHM but it addresses damage identification in rotating mechanical systems and the shared machinery used in power plant production. NonDestructive Evaluation (NDE) is an offline process localised to observe the damage. Moreover, a variety of pre-fabricated structures are monitored by the NDE, including rails and pressure containers.

In order to research the characteristics of a defined damage, NDE shall be applied and implemented within the framework identifying potential damage sites, including only the preliminary details on a damaged site. In comparison to the structure system, Statistical Process Control (SPC) is seen as a process-driven technology, where a broad range of sensors are used to detect process variations that can provide structural damage-related information.

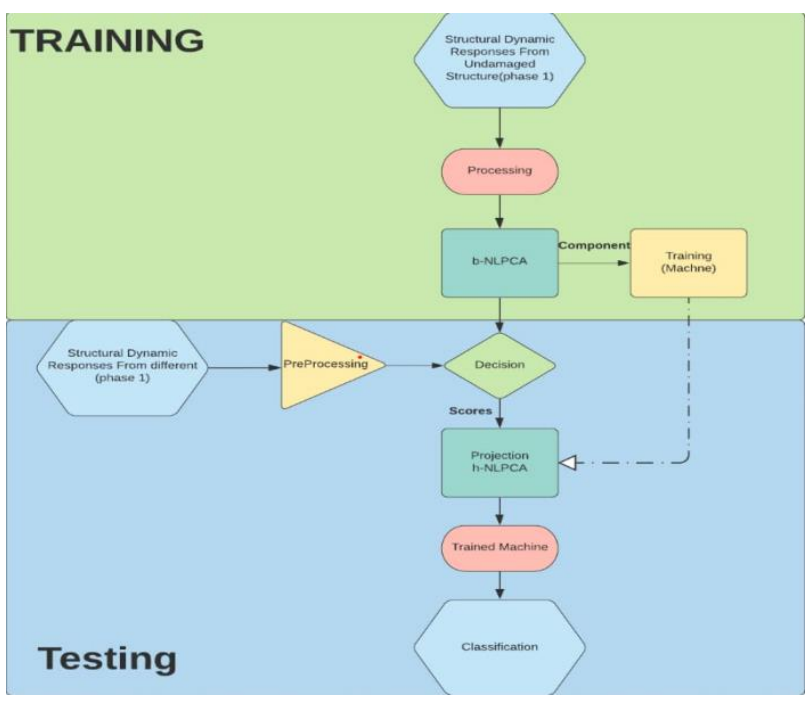

Fig. 1. Structural Dynamic responses [25].

\subsection{Deep convolutional neural network with transfer learning on SHM}

Water flow causes concrete surfaces to break down, resulting in spalling and rebar exposure. In order to protect the infrastructure, it is important that such damage should be detected. An extremely accurate damage detection strategy using a deep convolution neural network with transfer learning network is being introduced by this purpose. Secondly, photos using a high-definition camera with image expansion tool is also 
processed [26-27]. To identify damage Deep neural convolution network with hydro-connection learning transmission was introduced. Figure 2 explains the convolution process. The dataset comprises five distinct types of labels: crack, drain, spall, intact exposure and rebar. The Inception-v3 network is the core network, and the Inception-v3 network enhances the picture functionality [28-29]. Transfer learning is especially suitable for small datasets simultaneously. As a result, Inception-v3 and transfer learning have been introduced to detect damage to the water retaining infrastructure. The detection accuracy of model is higher than the SVM; the accuracy with process is about $96 \%$.

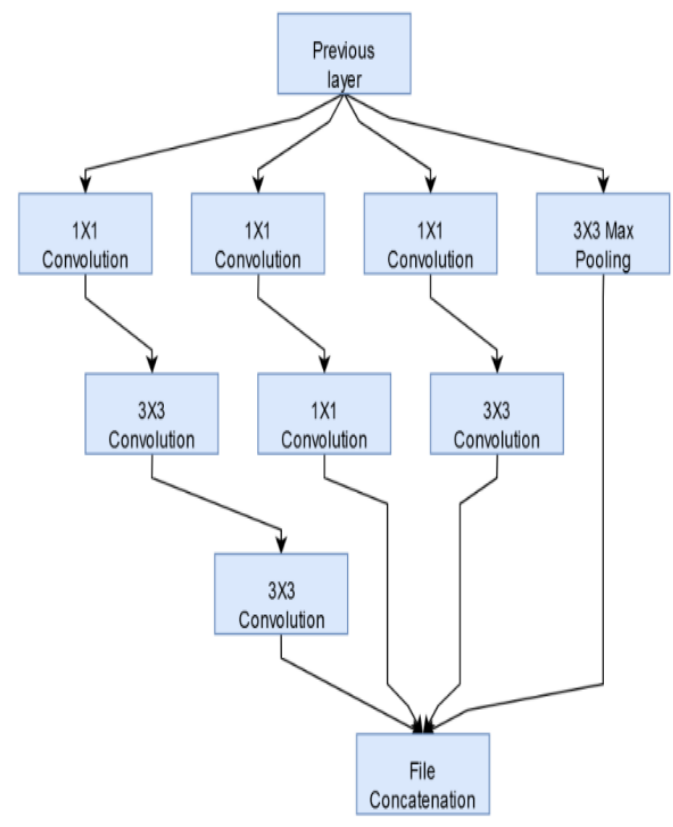

Fig. 2. Convolution process [29].

\subsection{Deep learning-based data anomaly detection method for structural health monitoring}

A large number of data is generated with the wider use of advanced SHM systems for civil infrastructure. Therefore, the analysis and mining of SHM data have become hot topics in the field of civil engineering [3031]. The dynamic environment of civil infrastructure systems, however, leads to several anomalies that contaminate the data. It severely affect the performance of the data analysis. This is one of the main obstacles to automatic real-time alerts because it is difficult to differentiate structural damage from the normalities associated with false results. Current data cleaning methods rely mainly on noise cleaning, although the incorrect detection of information takes a lot of expertise and time. Inspired by a real-world manual inspection procedure, SHM provides a machine vision and an anomaly system for profound knowledge with Deep neural visualisation networks, construction and anomaly recognition training [32-34]. This approach imitates the biological and ethical reasoning of human beings.
During visualisation, time series signals are converted into image vectors that are partly tracked in grey images. In the second level, a training data set consisting of randomly selected vectors is manually integrated into a deep neural network or a deep-neural network cluster, which is trained by techniques called automatically stacked encoders and greedy layer specific training. Deep neural networks can be used to detect potential anomalies in large quantities of non-regulated structural health monitoring data [35-37]. In order to illustrate the training procedure and to monitor the performance of the solution proposed, acceleration data are used using a genuinely long-term structural health monitoring system. The results show that data multi-pattern abnormalities can be detected automatically with high precision with computer vision and a comprehensive approach to the detection of anomalies in SHM systems. The time-series data in SHM are initially converted to a recognisable image in a machine and grey image vectors are used as a Deep Neural Network (DNN) training collection by imitating the human expert. The DNN is developed and trained by the technique of greedy layer training.

In order to confirm the viability and accuracy of the planned and trained DNN, acceleration data of the longdistance SHM can be measured. Training process of datatset is explained in Figure no. 3. Data abnormality and sensor counting results are distributed which are useful for the detailed cleaning and maintenance of the SHM system [38-40]. In comparison to the manual inspection system, computer vision and approach based on deep learning are significantly better. In the processing of SHM data, the method is new and critical both for the automatic real-time monitoring and alertness of SHM systems and for off-line data analysis. This article concentrates only on acceleration data, but it can also be used for other sensor types. The unattended analysis of anomalous photographs should be given greater attention in future work to eliminate manual interference. The multi-label method of classification is also applicable in the data measured in the SHM systems for competitor anomalies [41-43]. 


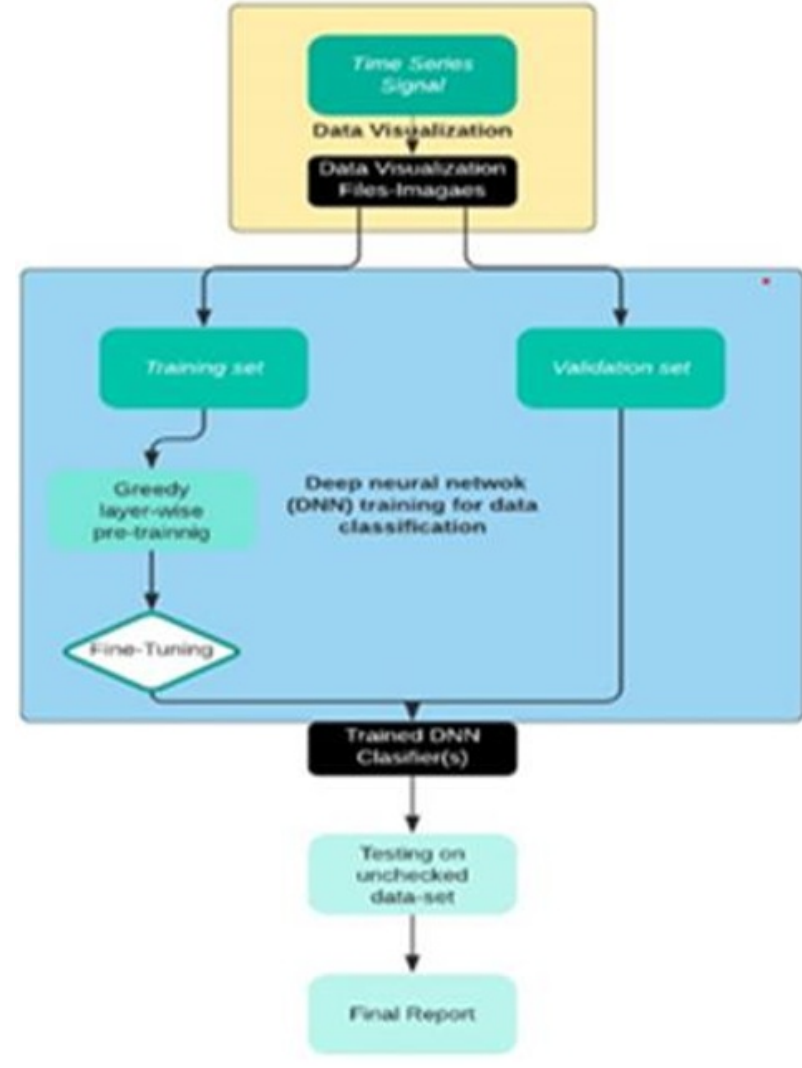

Fig. 3. Training process of dataset [35].

\section{Results and Discussion}

This paper provides an overview of R\&D in the field of civil infrastructure structural monitoring. Following a thorough analysis of the hypotheses, methods, innovations and implementations for sensors, the following inference was drawn: Sensors were commonly used due to their special merits. Monitoring of civil structures such as bridges, dams, pipelines, wind turbines, railway projects and life-cycle structures requires, measuring a broad variety of data including pressures, levels, accelerations etc. The implementation guidelines for sensor safety is of prime importance due to cracking and corrosion of concrete. In the area of early age strength control, impact identification and assessment as well as structural health monitoring for concrete structures, an advanced multifunctional intelligent unit is required at large scale. The early age tracking leads to damage index matrices in order to extract information on damage time and location. Structural health monitoring evaluations demonstrate that vital damages may be observed faster than conventional monitoring techniques. It is also possible to accurately assess the severity of cracks and the location of the damages.

\section{References}

1. Chathurdara Sri Nadith Pathirage et al., "Structural Damage Identification Based on Autoencoder Neural Networks and Deep Learning," Engineering
Structures 172, no. April (2018): 13-28, https://doi.org/10.1016/j.engstruct.2018.05.109.

2. Alberto Diez et al., "A Clustering Approach for Structural Health Monitoring on Bridges," Journal of Civil Structural Health Monitoring 6, 3, 429-45, (2016).

3. Hossein Babajanian Bisheh et al., "Damage Detection of a Cable-Stayed Bridge Using Feature Extraction and Selection Methods," Structure and Infrastructure Engineering 15, 9, 1165-77,(2019).

4. Yuequan Bao et al., "Computer Vision and Deep Learning-Based Data Anomaly Detection Method for Structural Health Monitoring," Structural Health Monitoring 18, 2, 401-21,(2019).

5. Ebrahim Nazarian et al., "Machine-Learning-Based Approach for Post Event Assessment of Damage in a Turn-of-the-Century Building Structure," Journal of Civil Structural Health Monitoring 8, 2, 23751,(2018) .

6. Giacomo Vincenzo Demarie and Donato Sabia, "A Machine Learning Approach for the Automatic Long-Term Structural Health Monitoring," Structural Health Monitoring 18, 3, 819-37, (2019) .

7. Radia Belkeziz and Zahi Jarir, "A Survey on Internet of Things Coordination," Proceedings 2016 3rd International Conference on Systems of Collaboration, SysCo 2016, 3, 619-35,(2017).

8. Aranud Deraemaeker et al., "New Trends in Vibration Based Structural Health Monitoring," International Centre for Mechanical Sciences, 520, 1-643,(2010).

9. Rafaelle Piazzaroli Finotti, Alexandre Abrahão Cury, and Flávio de Souza Barbosa, "An SHM Approach Using Machine Learning and Statistical Indicators Extracted from Raw Dynamic Measurements," Latin American Journal of Solids and Structures 16, 2, 1-17,(2019).

10. Ahmed Ibrahim et al., "A Machine Learning Approach for Structural Health Monitoring Using Noisy Data Sets," IEEE Transactions on Automation Science and Engineering 17, 2, 900908,(2020).

11. William Nick et al., "A Study of Machine Learning Techniques for Detecting and Classifying Structural Damage," International Journal of Machine Learning and Computing 5, 4, 313-18, (2015).

12. P. Gardner, R. J. Barthorpe, and C. Lord, "The Development of a Damage Model for the Use in Machine Learning Driven SHM and Comparison with Conventional SHM Methods," Proceedings of ISMA 2016 - International Conference on Noise and Vibration Engineering and USD2016 - International Conference on Uncertainty in Structural Dynamics, 3, 3333-46, (2016).

13. A. E. Aktan, A. J. Helmicki, and V. J. Hunt, "Issues in Health Monitoring for Intelligent Infrastructure," Smart Materials and Structures 7, 5, 674-92, (1998). 
14. Dongho Kang and Young Jin Cha, "Autonomous UAVs for Structural Health Monitoring Using Deep Learning and an Ultrasonic Beacon System with Geo-Tagging," Computer-Aided Civil and Infrastructure Engineering 33, 10, 885-902, (2018).

15. A. C. Neves et al., "Structural Health Monitoring of Bridges: A Model-Free ANN-Based Approach to Damage Detection," Journal of Civil Structural Health Monitoring 7, 5, 689-702, (2017).

16. Daniel Frank Hesser and Bernd Markert, "Excitation Strategies for Vibration Based Damage Detection Using Piezoelectric Transducers and Machine Learning," Pamm 16, 1, 141-42, (2016).

17. Vicente Lopes et al., "Impedance-Based Structural Health Monitoring with Artificial Neural Networks," Journal of Intelligent Material Systems and Structures 11, 3, 206-14, (2000).

18. Mohammad Hossein Rafiei and Hojjat Adeli, "A Novel Machine Learning-Based Algorithm to Detect Damage in High-Rise Building Structures," Structural Design of Tall and Special Buildings 26, 18, 1-11, (2017).

19. Mohammad Hossein Rafiei and Hojjat Adeli, "A Novel Unsupervised Deep Learning Model for Global and Local Health Condition Assessment of Structures," Engineering Structures,56,598-607, (2018).

20. Yunzhu Chen and Xingwei Xue, "Advances in the Structural Health Monitoring of Bridges Using Piezoelectric Transducers," Sensors (Switzerland) 18,12,1-24, (2018).

21. Diego A. Tibaduiza Burgos et al., Damage Identification in Structural Health Monitoring: A Brief Review from Its Implementation to the Use of Data-Driven Applications, Sensors (Switzerland), 20,10, (2020).

22. Andrea E Del Grosso, "Structural Health Monitoring: Research and Applications," Key Engineering Materials, 558, 13, (2013).

23. K Smarsly and D Hartmann, "Artificial Intelligence in Structural Health Monitoring," The Third International Conference on Structural Engineering, Mechanics and Computation. Cape Town, South Africa, 1, 705-10, (2007).

24. M. Azarbayejani et al., "A Probabilistic Approach for Optimal Sensor Allocation in Structural Health Monitoring," Smart Materials and Structures, 17, 5, (2008).

25. Sandeep Sony, Shea Laventure, and Ayan Sadhu, "A Literature Review of Next-Generation Smart Sensing Technology in Structural Health Monitoring," Structural Control and Health Monitoring 26, 3, 1-22, (2019).

26. Onur Avci et al., "Structural Damage Detection in Real Time: Implementation of 1D Convolutional Neural Networks for SHM Applications," Conference Proceedings of the Society for Experimental Mechanics Series, 10, 49-54, (2017).
27. Chuncheng Feng et al., "Structural Damage Detection Using Deep Convolutional Neural Network and Transfer Learning," KSCE Journal of Civil Engineering 23, 10, 4493-4502, (2019).

28. Ramin Ghiasi, Peyman Torkzadeh, and Mohammad Noori, "A Machine-Learning Approach for Structural Damage Detection Using Least Square Support Vector Machine Based on a New Combinational Kernel Function," Structural Health Monitoring 15, 3, 302-16, (2016).

29. Diego Tibaduiza et al., "A Damage Classification Approach for Structural Health Monitoring Using Machine Learning," Complexity, 18, 5, (2018).

30. Alvaro Araujo et al., "Wireless Measurement System for Structural Health Monitoring with High Time-Synchronization Accuracy," IEEE Transactions on Instrumentation and Measurement 61, 3, 801-10, (2012) .

31. X. W. Ye, C. Z. Dong, and T. Liu, "A Review of Machine Vision-Based Structural Health Monitoring: Methodologies and Applications," Journal of Sensors, 16, 8, (2016).

32. Ting Hua Yi, Hong Nan Li, and Xu Dong Zhang, "A Modified Monkey Algorithm for Optimal Sensor Placement in Structural Health Monitoring," Smart Materials and Structures, 21,10, (2012).

33. Xia Zhao et al., "Designing Strategy for MultiAgent System Based Large Structural Health Monitoring," Expert Systems with Applications 34, 2 1154-68, (2008).

34. Everaldo S. De Freitas et al., "Equivalent Circuit of Piezoelectric Diaphragms for Impedance-Based Structural Health Monitoring Applications," IEEE Sensors Journal 17, 17, 5537-46, (2017).

35. Majdi Flah et al., "Machine Learning Algorithms in Civil Structural Health Monitoring: A Systematic Review," Archives of Computational Methods in Engineering, 4, 9, (2020),

36. X. W. Ye, C. Z. Dong, and T. Liu, "A Review of Machine Vision-Based Structural Health Monitoring: Methodologies and Applications," Journal of Sensors, 16, 8, (2016).

37. Ting Hua Yi, Hong Nan $\mathrm{Li}$, and Xu Dong Zhang, "A Modified Monkey Algorithm for Optimal Sensor Placement in Structural Health Monitoring," Smart Materials and Structures , 21,10, (2012).

38. Xia Zhao et al., "Designing Strategy for MultiAgent System Based Large Structural Health Monitoring," Expert Systems with Applications 34, 2 1154-68, (2008)

39. Everaldo S. De Freitas et al., "Equivalent Circuit of Piezoelectric Diaphragms for Impedance-Based Structural Health Monitoring Applications," IEEE Sensors Journal 17, 17, 5537-46, (2017).

40. Majdi Flah et al., "Machine Learning Algorithms in Civil Structural Health Monitoring: A Systematic Review," Archives of Computational Methods in Engineering, 4, 9, (2020),. 
41. Jaime Vitola et al., "Structural Damage Detection and Classification Based on Machine Learning Algorithms," 8th European Workshop on Structural Health Monitoring, EWSHM 2016,4,2853-62, (2016).

42. Michel J. Murillo et al., "A Low-Cost Approach to Monitoring the Structural Health of Pedestrian Bridges," Espacios,40, 27 , (2019).

43. Bibin Kurian and Ranjith Liyanapathirana, Proceedings of the International Conference on ELearning, ICEL, Proceedings of the International Conference on E-Learning, ICEL, 2018, 10, (2018). 